Research, Society and Development, v. 10, n. 2, e9110212299, 2021

(CC BY 4.0) | ISSN 2525-3409 | DOI: http://dx.doi.org/10.33448/rsd-v10i2.12299

\title{
Cardiotoxicidade associada à terapia quimioterápica oncológica: Identificação dos
}

\section{fatores de risco}

\author{
Cardiotoxicity associated with oncological chemotherapy: Identification of risk factors \\ Cardiotoxicidad asociada con la quimioterapia oncológica: Identificación de factores de riesgo
}

Recebido: 23/01/2021 | Revisado: 24/01/2021 | Aceito: 27/01/2021 | Publicado: 06/02/2021

\author{
Paloma Geralda Mizael de Paula Silva \\ ORCID: https://orcid.org/0000-0002-0167-0861 \\ Universidade do Estado do Rio de Janeiro, Brasil \\ E-mail: palomamizael@yahoo.com.br \\ Vanessa Galdino de Paula \\ ORCID: https://orcid.org/0000-0002-7147-5981 \\ Universidade do Estado do Rio de Janeiro, Brasil \\ E-mail: vanegalpa@gmail.com \\ Luana Ferreira de Almeida \\ ORCID: https://orcid.org/0000-0001-8433-4160 \\ Universidade do Estado do Rio de Janeiro, Brasil \\ E-mail: luana.almeida3011@gmail.com \\ Eloá Carneiro Carvalho \\ ORCID: https://orcid.org/0000-0002-1099-370X \\ Universidade do Estado do Rio de Janeiro, Brasil \\ E-mail: eloacarvalhogrossi@gmail.com \\ Thereza Christina Mó y Mó Loureiro Varella \\ ORCID: https://orcid.org/0000-0001-9389-1161 \\ Universidade do Estado do Rio de Janeiro, Brasil \\ Email: thereza1208@gmail.com \\ Samira Silva Santos Soares \\ ORCID: https://orcid.org/0000-0001-9133-7044 \\ Universidade do Estado do Rio de Janeiro, Brasil \\ E-mail: samira_opg@hotmail.com \\ Norma Valéria Dantas de Oliveira Souza \\ ORCID: https://orcid.org/0000-0002-2936-3468 \\ Universidade do Estado do Rio de Janeiro, Brasil \\ E-mail: norval_souza@yahoo.com.br \\ Sandra Regina Maciqueira Pereira \\ ORCID: https://orcid.org/0000-0002-0550-2494 \\ Universidade do Estado do Rio de Janeiro, Brasil \\ E-mail: sandregina@gmail.com \\ Karla Biancha Silva de Andrade \\ ORCID: https://orcid.org/0000-0002-6216-484X \\ Universidade do Estado do Rio de Janeiro, Brasil \\ E-mail: karla.biancha@gmail.com
}

\begin{abstract}
Resumo
Objetivo: Identificar os fatores de risco para cardiotoxicidade nos pacientes oncológicos em tratamento antineoplásicos. Metodologia: revisão integrativa da literatura, realizada nas bases de dados MEDLINE/PUBMED, SCIELO E BVS por meio do cruzamento dos descritores Neoplasias, Protocolos Clínicos, Fatores de Risco e Cardiotoxicidade e seus respectivos entretermos interligados pelos operadores boleanos "AND" e"OR", com um recorte temporalde 2017 a 2019.Resultados:após leitura e análise das obras (n: 30) encontradas surgiram sete categorias: sobrepeso e obesidade; arritmias cardíacas; doença preexistente, irradiação mediastinal, predisposição genética; inatividade física; hipertensão arterial sistólica, diabetes, dislipidemia e tabagismo; tempo longo da terapia e dose cumulativa; associação das classes dos quimioterápicos. Conclusão: muitos fatores de risco estão presentes nos pacientes oncológicos, contribuindo para o desenvolvimento da cardiotoxicidade. Dessa maneira, é essencial que a enfermagem oncológica tenha em sua prática foco na identificação precoce dos mesmos a fim de prevenir e controlar a cardiotoxicidade.
\end{abstract}

Palavras-chave: Neoplasias; Protocolo clínico; Fatores de risco; Cardiotoxicidade. 
Research, Society and Development, v. 10, n. 2, e9110212299, 2021

(CC BY 4.0) | ISSN 2525-3409 | DOI: http://dx.doi.org/10.33448/rsd-v10i2.12299

\begin{abstract}
Objective: To identify the risk factors for cardiotoxicity in cancer patients undergoing antineoplastic treatment. Methodology: Integrative literature review, performed in the MEDLINE/PUBMED, SCIELO AND VHL databases, by crossing the descriptors: neoplasms, clinical protocols, risk factors and cardiotoxicity and their respective interconnected terms by the Boolean operators "AND" and "OR", with a time frame from 2017 to 2019). Results: After reading and analyzing the works (n: 30) found, seven categories emerged: Overweight and obesity; cardiac arrhythmias; preexisting disease, mediastinal irradiation, genetic predisposition; physical inactivity; systolic arterial hypertension, diabetes, dyslipidemia and smoking; long therapy time and cumulative dose; association of chemotherapy classes. Conclusion: Many risk factors are present in cancer patients, thus contributing to the development of cardiotoxicity. Accordingly, it is essential that oncological nursing has, in its practice, focus on early identification of them, in order to prevent and control cardiotoxicity.
\end{abstract}

Keywords: Neoplasms; Clinical protocol; Risk factors; Cardiotoxicity.

\title{
Resumen
}

Objetivo: Identificar los factores de riesgo de cardiotoxicidad en pacientes oncológicos sometidos a tratamiento antineoplásico. Metodología: Revisión integradora de la literatura, efectuada en las bases de datos MEDLINE/PUBMED, SCIELO y BVS, mediante el cruce de los descriptores: neoplasias, protocolos clínicos, factores de riesgo y cardiotoxicidad y sus respectivos términos interconectados por los operadores boléanos “AND” y “OR”, con un corte temporal de 2017 a 2019. Resultados: Después de la lectura y el análisis de los trabajos (n: 30), surgieron siete categorías: sobrepeso y obesidad; arritmias cardíacas; enfermedad preexistente, irradiación mediastínica, predisposición genética; inactividad física; hipertensión sistólica, diabetes, dislipidemia y tabaquismo; largo tiempo de terapia y dosis acumulada; asociación de clases quimioterápicas. Conclusión: Hay muchos factores de riesgo en los pacientes con cáncer que contribuyen al desarrollo de la cardiotoxicidad. Así, es fundamental que la enfermería oncológica tenga, en su práctica, enfoque en su identificación precoz, con miras a prevenir y controlar la cardiotoxicidad.

Palabras clave: Neoplasias; Protocolo clínico; Factores de riesgo; Cardiotoxicidad.

\section{Introdução}

A mortalidade de pacientes acometidos pelas doenças crônicas não transmissíveis soma uma totalidade de aproximadamente 36 milhões de mortes, representando uma porcentagem de $63 \%$ de óbitos em todo o mundo. Dentre as principais causas de falecimentos estãoas doenças cardiovasculares (48\%), doenças oncológicas (21\%), doenças respiratórias crônicas (12\%) e diabetes (3\%) (Folha informativa - Câncer, 2018).

No que se refere à doença oncológica, a estimativa para o Brasil no triênio de 2020-2022 aponta a ocorrência de 625 mil casos novos de câncer, o de pele não melanoma previsto para 177 mil casos novos, seguido pelo câncer de mama e próstata, com 66 mil cada, o de cólon e reto 41 mil, pulmão 30 mil e estômago 21 mil. Estimativa 2020: incidência de câncer no Brasil(Instituto Nacional de Câncer 2019).

Atualmente são instituídos diversos tipos de tratamento para a doença oncológica, sendo as mais tradicionais a cirurgia, a radioterapia e a quimioterapia convencional. Além dessas modalidades, existe a terapia molecularmente direcionada e imunoterapia, essas duas últimas são mais recentes (ABC do câncer: abordagens básicas para o controle do câncer, 2020).

O cenário das doenças oncológicas vem se transformando e devido aos avanços da terapia antineoplásica houve um aumento na sobrevida das pessoas acometidas por essa doença. No entanto, associado a essa mudança o risco para o desenvolvimento de doenças cardiovasculares relacionados à toxicidade da droga quimioterápica se torna mais comum nessa população (Departamento de Atenção Básica, 2013).

A cardiotoxicidade é uma das mais graves toxidades e pode se apresentar de três formas: aguda, subaguda e crônica. As formas aguda e subaguda podem surgir até 14 dias após o término do tratamento, é representada pelas alterações na repolarização dos ventrículos e no intervalo Q-T, arritmia supra e ventriculares, síndromes coronarianas agudas, pericardite e miocardite. A forma crônica inclui dois subtipos, varia de acordo com o início dos sintomas clínicos, o primeiro subtipo acontece em um período de 12 meses após o término do tratamento quimioterápico e o segundo após 12 meses do final do 
Research, Society and Development, v. 10, n. 2, e9110212299, 2021

(CC BY 4.0) | ISSN 2525-3409 | DOI: http://dx.doi.org/10.33448/rsd-v10i2.12299

tratamento. A manifestação mais comum da cardiotoxicidade crônica é a insuficiência cardíaca, podendo levar à morte(I Diretriz Brasileira de Cardio-Oncologia da Sociedade Brasileira de Cardiologia, 2020).

Os efeitos cardiotóxicos possuem relação direta com a dose e velocidade de infusão da droga, estando relacionados também com a associação das droga a ntineoplásicas, insuficiência hepática e renal. A disfunção ventricular sistólica e diastólica assintomática ou sintomática varia e é mais frequente em pacientes com fatores de risco já bem fundamentados, como extremos de idade, disfunção ventricular prévia, hipertensão arterial, diabetes, uso de associação de quimioterápicos, radioterapia mediastinal e suscetibilidade genética. Esses fatores de riscos associados com as drogas cardiotóxicas podem levar à agressão miocárdica, disfunção sistólica e insuficiência cardíaca (Diretriz Brasileira de Cardio-Oncologia da Sociedade Brasileira de Cardiologia, 2020).

A fim de conhecer os fatores de risco que podem induzir a toxicidade cardíaca, este estudo teve por objetivo identificar, a partir da literatura científica, fatores de risco para a cardiotoxicidade nos pacientes oncológicos em tratamento.

\section{Metodologia}

Trata-se de um estudo do tipo revisão integrativa de literatura, direcionado para o levantamento dos fatores de riscos em pacientes com diagnósticos de câncer e em tratamento oncológico, com enfoque na cardiotoxicidade. Com base no objetivo apresentado, priorizou-se a análise a partir de uma perspectiva qualitativa, sendo descrita por Köche (2011) como aquela que não pode ser quantificada, os fatos podem ser determinados pela experiência e percepção sensível. Corroborando, para Pereira et al. (2018), na pesquisa qualitativa é importante a interpretação do pesquisador com suas opiniões sobre os fenômenos estudados.

A pergunta que direcionou o estudo foi formulada por meio da estratégia PIO, representada por um acrônimo cuja letra $\mathrm{P}$ relaciona-se ao paciente, a letra I à intervenção e Ooctomes (desfecho/ resultados). Esse método orienta o pesquisador para uma melhor busca da literatura disponível de forma completa e eficaz. A partir disso, adequando ao estudo em questão o caractere P: paciente oncológico; I: tratamento antineoplásico; O: Cardiotoxicidade, o que originou a seguinte pergunta: quais os fatores de riscos para o desenvolvimento de cardiotoxicidade nos pacientes oncológicos em tratamento antineoplásicos?

Para este estudo, realizou-se a elaboração do conteúdo embasado em evidências científicas. Depois de estabelecidas a temática e adelimitação da questão norteadora, definiram-se os seguintes critérios de inclusão: artigos completos e disponíveis na base de dados; publicados no recorte temporal de 2017-2019; nas línguas portuguesa, inglesa e espanhola;e que possuíam como sujeito de pesquisa pacientes adultos. Excluíram-se editoriais, carta-resposta, monografia, documentos informativos, livros, dissertações e teses. A coleta de dados foi realizada no período de junho a setembro de 2020.

A estratégia de busca foi realizada utilizando os descritores e seus entretermos, referenciados pelos Descritores em Ciências da Saúde (DeCS) e Medical SubjectHeading(MeSH) definidos como "neoplasia”, "cardiotoxicidade”, "protocolos clínicos" "fatores de riscos". Realizou-se o cruzamento a partir dos operadores boleanos"and"e“or" afim de obter uma busca mais específica. Os termos foram correlacionados na seguinte estratégia final: (P) AND (I) AND (C) AND (O). A pesquisa foi realizada em publicações indexadas nas seguintes bases de dados: PublicMedline (PUBMED), na qual se obteve o resultado de 29 artigos;Biblioteca Virtual em Saúde (BVS), com 21 artigos; e ScientificElectronic Library Online (SCIELO),com 3 artigos.

Após a busca a busca realizada na base de dados e aplicação dos critérios de inclusão e exclusão, chegou-se a um total de 53 artigos, excluíram-se 30 de acordo com os critérios de elegibilidade, conforme ilustrado na Figura 1. 
Research, Society and Development, v. 10, n. 2, e9110212299, 2021

(CC BY 4.0) | ISSN 2525-3409 | DOI: http://dx.doi.org/10.33448/rsd-v10i2.12299

Figura 1 - Fluxograma de identificação e seleção dos estudos.

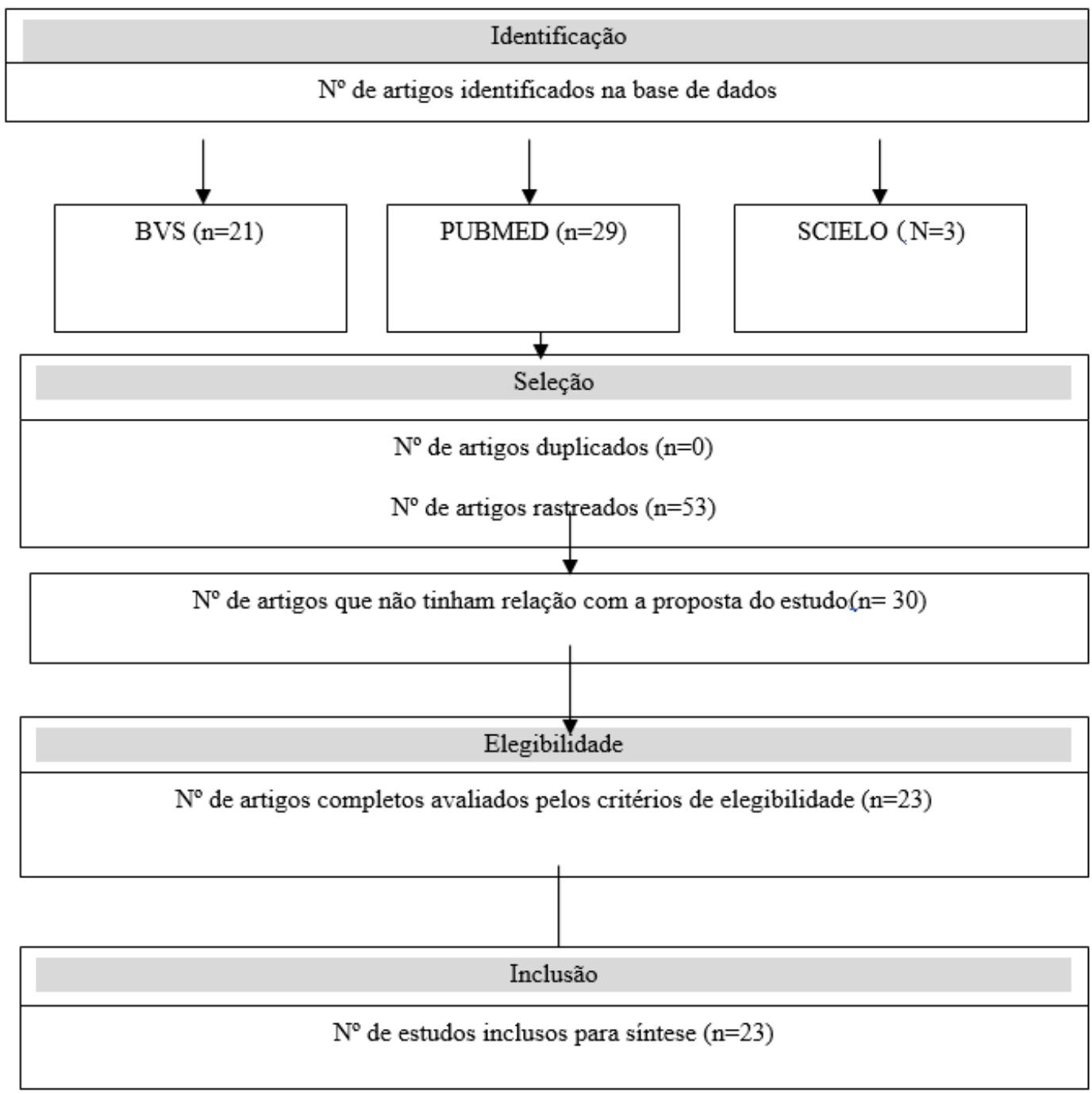

Fonte: autores.

A figura acima ilustra o trajeto percorrido de busca, a começar dos resultados iniciais por cada base de dados, até o quantitativo total de publicações encontradas após a aplicação do critério de inclusão e exclusão.

A terceira etapa compreendeu a elaboração de um método mais preciso e específico com o propósito de organizar os dados do estudo. Construiu-se uma tabela nosoftwareMicrosoft Office Excel 2010 com as seguintes variáveis: título do artigo; revista; ano de publicação; e principal percepção.

$\mathrm{Na}$ quarta etapa, os artigos selecionados foram lidos na íntegra de forma exaustiva, eas características do estudo foram sintetizadas e analisadas de forma narrativa.

Para apresentação e discussão dos resultados, optou-se pelacategorização da temática. Por se tratar de uma revisão de literatura, este estudo não necessitou da aprovação de Comitê de Ética em Pesquisa, entretanto foram considerados aspectos éticos no que diz respeito aos direitos autorais dos artigos selecionados.

\section{Resultados}

A amostra foi composta de 23 artigos, que foram distribuídos para análise, conforme o título, ano, autor, periódico e temática principal. 
Research, Society and Development, v. 10, n. 2, e9110212299, 2021

(CC BY 4.0) | ISSN 2525-3409 | DOI: http://dx.doi.org/10.33448/rsd-v10i2.12299

Quadro 1 - Distribuição dos artigos selecionados para análise segundo título, ano, autor, periódico e temática principal.

\begin{tabular}{|c|c|c|c|c|c|}
\hline & TÍTULO & ANO & AUTOR & PERIÓDICO & TEMÁTICA PRINCIPAL \\
\hline 1 & $\begin{array}{l}\text { Association of body mass index and cardiotoxicity related to } \\
\text { anthracyclines and trastuzumab in early breast cancer: } \\
\text { French CANTO cohort study }\end{array}$ & 2019 & Kabore & Plos Medicine & $\begin{array}{l}\text { O grupo obeso foi mais propenso à cardiotoxicidade do que o grupo com peso } \\
\text { hormal.A obesidade pode estar associada ao aumento significativo da cardiotoxicidade. }\end{array}$ \\
\hline 2 & $\begin{array}{l}\text { Anthracycline Chemotherapy-Induced Cardiotoxicity } \\
\text { in Breast Cancer Survivors:A SystematicReview }\end{array}$ & 2019 & Lin & OncolNursForum & $\begin{array}{l}\text { Antraciclinas podem trazer prejuízo para o funcionamento sistólico e diastólico de } \\
\text { ventrículo esquerdo e ventrículo direito, condução elétrica atrial prejudicada, } \\
\text { miopatia e insuficiência cardíaca. } \\
\text { Principais fatores de risco foram dose cumulativa de antraciclina, terapia prévia com } \\
\text { transtuzumabe, hipertensão, aumento do índice de massa corporal, área de superfície } \\
\text { corporal e idade. }\end{array}$ \\
\hline 3 & $\begin{array}{l}\text { Impact of exercise training on cardiotoxicity and cardiac } \\
\text { health outcomes in women with breast cancer anthracycline } \\
\text { chemotherapy: a study protocol for a randomized controlled } \\
\text { trial. }\end{array}$ & 2019 & Antunes & Trials, & $\begin{array}{l}\text { Estudo prospectivo. O exercício físico foi proposto como uma ferramenta protetora } \\
\text { em pacientes com câncer de mama que utilizam as antraciclinas como } \\
\text { tratamento,embora permaneça incerto, existem mais estudos em animais do que em } \\
\text { humanos. }\end{array}$ \\
\hline 4 & $\begin{array}{l}\text { Role of Speckle Tracking Echocardiography in the } \\
\text { Evaluation of Breast } \\
\text { Cancer Patients Undergoing Chemotherapy: Review and } \\
\text { Meta-analysisoftheLiterature }\end{array}$ & 2019 & Bergamini & Cardiovascular Toxicology & $\begin{array}{l}\text { A técnica de imagem cardíaca auxilia na detecção precoce e subclínica de lesões } \\
\text { cardíacas, servindo de auxilio no manejo dos profissionais da área da cardio- } \\
\text { oncologia. }\end{array}$ \\
\hline 5 & $\begin{array}{l}\text { Persistent Impairment in Cardiopulmonary Fitness after } \\
\text { Breast Cancer Chemotherapy. }\end{array}$ & 2019 & Foulkes & $\begin{array}{l}\text { Medicine \& Science In } \\
\text { Sports \& Exercise }\end{array}$ & $\begin{array}{l}\text { Redução da função cardíaca após o exercício físico pode indicar precocemente } \\
\text { comprometimento cardíaco e risco aumentado de insuficiência cardíaca após } \\
\text { tratamento quimioterápico. Pacientes que fazem exercícios físicos obtêm melhores } \\
\text { resultados funcionais, principalmente após o tratamento, pois o comprometimento } \\
\text { cardíaco surgiu mais tardiamente. A quimioterapia está associada à diminuição da } \\
\text { aptidão cardiopulmonar que coincide com um prejuízo tardio da função cardíaca. }\end{array}$ \\
\hline 6 & $\begin{array}{l}\text { One year versus a shorter duration of adjuvant trastuzumab } \\
\text { for HER2- positive early breast cancer: a systematic review } \\
\text { and meta-analysis }\end{array}$ & 2018 & Inno & $\begin{array}{l}\text { Breast Cancer Research and } \\
\text { Treatment }\end{array}$ & $\begin{array}{l}\text { Não há nenhum benefício negativo significativo do tratamento com a duração de } 1 \\
\text { ano com transtuzumabe em relação a um período mais curto de tratamento ( } 6 \text { meses) } \\
\text { em doença em estágio I, receptor de estrogênio-positivo e linfonodo negativo, } \\
\text { pacientes de baixo risco ou alto risco de toxicidade cardíaca. } \\
\text { A redução mais curta com transtuzumabe está associada à diminuição significativa do } \\
\text { risco de eventos cardíacos. }\end{array}$ \\
\hline 7 & $\begin{array}{l}\text { Early diastolic strain rate measurements by cardiac MRI in } \\
\text { breast cancer patients treated with trastuzumab: a }\end{array}$ & 2018 & Gong & International Journal & $\begin{array}{l}\text { Não existe correlação consistente entre a taxa de esforço diastólico e a redução da } \\
\text { fração de ejeção. As frações de ejeção ventricular têm maior potencial para identificar }\end{array}$ \\
\hline
\end{tabular}


Research, Society and Development, v. 10, n. 2, e9110212299, 2021

(CC BY 4.0) | ISSN 2525-3409 | DOI: http://dx.doi.org/10.33448/rsd-v10i2.12299

\begin{tabular}{|c|c|c|c|c|c|}
\hline & longitudinal study. & & & Cardiovascular Imaging & os primeiros sinais de lesão miocárdica. \\
\hline 8 & $\begin{array}{l}\text { Cardiovascular Toxicities with Vascular Endothelial Growth } \\
\text { Factor Receptor Tyrosine Kinase Inhibitors in Cancer } \\
\text { Patients: A Meta-Analysis of } 77 \text { Randomized Controlled } \\
\text { Trials. }\end{array}$ & 2018 & $: \mathrm{Li}$ & Clinical Drug Investigation & $\begin{array}{l}\text { A hipertensão e disfunção cardíaca foram um dos efeitos cardiovasculares mais } \\
\text { comuns encontrados nos pacientes em uso de inibidores de tirosina quinase do } \\
\text { receptor. }\end{array}$ \\
\hline 9 & $\begin{array}{l}\text { The incidence of atrial fibrillation with trastuzumab } \\
\text { treatment: A systematic review and meta-analysis. }\end{array}$ & 2018 & Yuan & Cardiovascular Therapeutics & $\begin{array}{l}\text { A fibrilação atrial pode sermais facilmente encontrada em pacientes mais velhos por } \\
\text { serem mais sintomáticos em relação aos jovens, portanto mais propensos a } \\
\text { descontinuar a terapia com trastuzumabe. A associação do trastuzumabe com } \\
\text { antraciclinas pode provocar cardiotoxicidade com insuficiência cardíaca sintomática } \\
\text { grave e disfunção cardíaca em imagens ecocardiográficas. }\end{array}$ \\
\hline 10 & $\begin{array}{l}\text { Trastuzumab in Female Breast Cancer Patients With } \\
\text { Reduced Left Ventricular Ejection Fraction. }\end{array}$ & 2018 & Nowsheen & $\begin{array}{l}\text { Of the American Heart } \\
\text { Association }\end{array}$ & $\begin{array}{l}\text { O uso do trastuzumabe pode ser considerado em pacientes com função cardíaca } \\
\text { reduzida, no entanto esses pacientes precisam ser acompanhados de perto e precisam } \\
\text { estar informados sobre os riscos cardíacos da terapia. Os sintomas de insuficiência } \\
\text { cardíaca ocorrem de forma mais frequentes em pacientes com a fração de ejeção } \\
\text { reduzida.Amortalidade tardia após o uso de trastuzumabe também foi mais frequente } \\
\text { nesse perfil de paciente. }\end{array}$ \\
\hline 11 & $\begin{array}{l}\text { Prevention of Cardiotoxicities With Traditional and Novel } \\
\text { Chemotherapeutic Agents. }\end{array}$ & 2018 & Sharalaya & $\begin{array}{lll}\text { Current } & \text { Heart } & \text { Failure } \\
\text { Reports } & & \end{array}$ & $\begin{array}{l}\text { O uso de terapia individualizada para cada paciente, o acesso a programas de cardio- } \\
\text { oncologia e detecção precoce dos efeitos colaterais cardíacos fazem parte da } \\
\text { terapiacardioprotetora. A limitação da dose de antraciclina para reduzir o risco de } \\
\text { cardiotoxicidade. }\end{array}$ \\
\hline 12 & $\begin{array}{l}\text { When and how to treat women with HER2-positive, small } \\
\text { (pT1a-b), node-negativebreastcancer? }\end{array}$ & 2018 & Gori & $\begin{array}{l}\text { Critical Reviews in } \\
\text { Oncology/hematology }\end{array}$ & $\begin{array}{l}\text { Houve uma redução significativa dos eventos adversos cardíacosem pacientes que } \\
\text { faziam quimioterapia com transtuzumabe em menos de } 1 \text { ano. O uso do } \\
\text { transtuzumabe em curto período pode ser indicado para pacientes com risco baixo de } \\
\text { recidiva e alto risco de cardiotoxicidade. } \\
\text { A redução da fração de ejeção e a insuficiência cardíacasão um dos efeitos } \\
\text { cardiotoxicicos. } \\
\text { O sobrepeso e obesidade são fatores de risco para cardiotoxicidade em pacientes que } \\
\text { fazem uso de transtuzumabe e antraciclinas }\end{array}$ \\
\hline 13 & $\begin{array}{l}\text { Chemotherapy-induced cardiotoxicity: new insights into } \\
\text { mechanisms, monitoring, and prevention }\end{array}$ & 2018 & Dessalvi & Of Cardiovascular Medicine & $\begin{array}{l}\text { Estratégias farmacológicas e não farmacológicas para prevenção e tratamento da } \\
\text { cardiotoxicidade. } \\
\text { Mudanças no estilo de vida e exercícios físicos, além de promover impacto em } \\
\text { fatores de riscos cardiovasculares, como hipertensão, dislipidemia, obesidade e } \\
\text { diabetes. O Dexrazoxano é um quelante de ferro configurado como um dos } \\
\text { melhores cardioprotetores. As estratégias farmacológicas incluem } \\
\text { betabloqueadores,bloqueados dos receptores de angiotensina II, estatinas, inibidores } \\
\text { da fosfodiesterase-5 e inibidor da enzima conversora de angiotensina. }\end{array}$ \\
\hline
\end{tabular}


Research, Society and Development, v. 10, n. 2, e9110212299, 2021

(CC BY 4.0) | ISSN 2525-3409 | DOI: http://dx.doi.org/10.33448/rsd-v10i2.12299

\begin{tabular}{|c|c|c|c|c|c|}
\hline 14 & $\begin{array}{l}\text { Everolimus as cancer therapy: Cardiotoxic or an unexpected } \\
\text { antiatherogenic agent? A narrativereview }\end{array}$ & 2018 & Karvelas & $\begin{array}{ll}\text { Hellenic } & \text { Journal of } \\
\text { Cardiology } & \end{array}$ & $\begin{array}{l}\text { O Everolimus provoca cardiotoxicidade levando a eventos coronários agudos, como } \\
\text { redução da FE, arritmias, sinais de insuficiência cardíaca aguda e reação pericárdica. } \\
\text { Alguns dos efeitos adversos mais encontrados foram a hiperglicemia, hiperlipidemiae } \\
\text { hipertensão. A droga não tem efeito cardiotóxico direto, porém influencia diretamente } \\
\text { nos fatores de risco cardiovasculares. }\end{array}$ \\
\hline 15 & $\begin{array}{l}\text { Effect of candesartan andmetoprolol on } \\
\text { myocardial tissue composition during } \\
\text { anthracycline treatment: the PRADA trial }\end{array}$ & 2017 & Heck & $\begin{array}{l}\text { European Heart Journal } \\
\text { Cardiovascular Imaging }\end{array}$ & $\begin{array}{l}\text { A cardiotoxicidade provocada por antraciclinas depende da dose e aumentou a fração } \\
\text { de extração de volume extravascular.Pacientes que receberam candesartan } \\
\text { experimentaram menos declínio na função sistólica do que os pacientes que não } \\
\text { receberam candesartan. }\end{array}$ \\
\hline 16 & $\begin{array}{l}\text { Pertuzumab, trastuzumab, and standard anthracycline- and } \\
\text { taxane-based chemotherapy for the neoadjuvant treatment of } \\
\text { patients with HER2-positive localized breast cancer } \\
\text { (BERENICE): a phase II, open-label, multicenter, } \\
\text { multinational cardiac safety study }\end{array}$ & 2018 & Swain & Annals of Oncology & $\begin{array}{l}\text { Quimioterapia contendo antraciclina comum para o tratamento neoadjuvante de } \\
\text { câncer de mama resultou em perfis cardíacos e de segurança geral e taxas de parada } \\
\text { cardiorrespiratória. }\end{array}$ \\
\hline 17 & $\begin{array}{l}\text { Cardiac biomarkers for early detection and prediction of } \\
\text { trastuzumab and/or lapatinib-induced cardiotoxicity in } \\
\text { patients with HER2-positive early-stage breast cancer: a } \\
\text { NeoALTTOsub-study (BIG 1-06) }\end{array}$ & 2017 & Ponde & $\begin{array}{l}\text { Breast Cancer Research And } \\
\text { Treatment, }\end{array}$ & $\begin{array}{l}\text { Troponinas e NT-proBNP são marcadores de lesão cardíaca.No estudo, o aumento } \\
\text { desses marcadores foi baixo, o que impossibilitou correlacionar com possíveis } \\
\text { eventos cardíacos. Hipótese sugere que a elevação dos biomarcadores está mais } \\
\text { relacionada ao uso de antraciclinas, trastuzumabe, lapatinibe ou sua combinação } \\
\text { sugere ser uma terapia segura no ponto de vista cardíaco. }\end{array}$ \\
\hline 18 & $\begin{array}{l}\text { Prospective evaluation of the cardiac safety of HER2- } \\
\text { targeted therapies in patients with HER2-positive breast } \\
\text { cancer and compromised heart function: the SAFE-HEaRt } \\
\text { study }\end{array}$ & 2019 & Lynce & $\begin{array}{l}\text { Breast Cancer Research And } \\
\text { Treatment }\end{array}$ & $\begin{array}{l}\text { Os pacientes com disfunção cardíaca tratados com terapia direcionada a } \\
\text { HER2associada à medicação cardioprotetora não desenvolveram efeitos colaterais, } \\
\text { possibilitando a continuação do tratamento oncológico e impacto na qualidade de } \\
\text { vida. }\end{array}$ \\
\hline 19 & $\begin{array}{l}11 \text { years' follow-up of trastuzumab after adjuvant } \\
\text { chemotherapy in HER2-positive early breast cancer: final } \\
\text { analysis of the HERceptin Adjuvant (HERA) trial }\end{array}$ & 2017 & Cameron & The Lancet & $\begin{array}{l}\text { Não foi observada diferença significante nos efeitos cardíacos primários relacionados } \\
\text { ao uso de transtuzumabe em } 1 \text { ano comparado a dois; já os efeitos cardíacos } \\
\text { secundários ocorreram com maior frequência no grupo que fez uso de } \\
\text { transtuzumabepor } 2 \text { anos. Em todos os grupos os desfechos cardíacos foram } \\
\text { diminuídos após a conclusão do tratamento do que no período de tratamento, e após } \\
\text { um longo tempo de finalização do tratamento. Não houve evidência de benefício de } \\
\text { longo prazo de } 2 \text { anos em comparação com } 1 \text { ano de trastuzumabe quando } \\
\text { administrado como tratamento sequencial após a quimioterapia. }\end{array}$ \\
\hline 20 & $\begin{array}{l}\text { Long-Term Follow-Up of Cardiac Function and Quality of } \\
\text { Life for Patients in NSABP Protocol B-31/NRG Oncology: } \\
\text { A Randomized Trial Comparing the Safety and Efficacy of } \\
\text { Doxorubicin and Cyclophosphamide (AC) Followed by } \\
\text { Paclitaxel With AC Followed by Paclitaxel and } \\
\text { Trastuzumab in Patients With Node-Positive Breast Cancer }\end{array}$ & 2017 & Ganz & Journal of Clinical Oncology & $\begin{array}{l}\text { Em mulheres que sobreviveram ao câncer de mama e que não possuem história } \\
\text { cardíaca prévia quando otrastuzumabe é incorporado na terapia adjuvante com } \\
\text { antraciclina, não tem repercussão cardíaca em longo prazo. }\end{array}$ \\
\hline
\end{tabular}


Research, Society and Development, v. 10, n. 2, e9110212299, 2021

(CC BY 4.0) | ISSN 2525-3409 | DOI: http://dx.doi.org/10.33448/rsd-v10i2.12299

\begin{tabular}{|l|l|l|l|l|l|}
\hline & $\begin{array}{l}\text { With Tumors Overexpressing Human Epidermal Growth } \\
\text { Factor Receptor 2 }\end{array}$ & & & \\
\hline 21 & $\begin{array}{l}\text { Nine weeks versus 1 year adjuvant trastuzumab in } \\
\text { combination with chemotherapy: final results of the phase } \\
\text { III randomized Short-HER study }\end{array}$ & 2018 & Conte & Annals of Oncology & $\begin{array}{l}\text { Terapia com trastuzumabe durante 9 semanas também pode contribuir na redução } \\
\text { significativa da recorrência do câncer de mama. Um tratamento com um período de } \\
\text { tempo mais curto está associado a uma menor toxicidade cardíaca. }\end{array}$ \\
\hline 22 & $\begin{array}{l}\text { Evaluation and management of chemotherapy-induced } \\
\text { cardiotoxicity in breast cancer: a Delphi study }\end{array}$ & 2016 & Gavila & $\begin{array}{l}\text { Clinical and Translational } \\
\text { Oncology }\end{array}$ & $\begin{array}{l}\text { O trastuzumabe está associado a um risco grande de induzir cardiotoxicidade em } \\
\text { longo prazo,quando associado às antraciclinas possui um risco ainda maior. A função } \\
\text { cardíaca deve ser avaliada antes do início do tratamento e acompanhada durante. } \\
\text { Fatores como hipertensão, idade, diabetes, obesidade, tabagismo, sedentarismo e } \\
\text { hipercolesterolemia também foram avaliados. }\end{array}$ \\
\hline 23 & $\begin{array}{l}\text { Development of anthracycline-induced dilated } \\
\text { cardiomyopathy due to mutation on LMNA gene in a breast } \\
\text { cancer patient: a case report }\end{array}$ & 2018 & Barros & $\begin{array}{l}\text { Arquivos Brasileiros de } \\
\text { Cardiologia }\end{array}$ & $\begin{array}{l}\text { O tratamento com antraciclinas pode ser considerado um fator de risco para } \\
\text { cardiotoxicidade e desenvolvimento precoce de cardiomiopatia dilatada devido à sua } \\
\text { predisposição genética }\end{array}$ \\
\hline
\end{tabular}

Fonte: Dados da pesquisa. 
O Quadro 1 expõe uma síntese dos estudos incluídos nesta revisão, evidenciando a temática, ano, autor e temática principal de cada estudo. Essas variáveis foram utilizadas como ferramenta para embasamento na construção da discussão corroborando achados de outros autores que abordaram a mesma temática

A análise dos estudos conduziu a elaboração de sete categorias relacionadas aos fatores de riscos e à classe dos quimioterápicos: 1 - Sobrepeso e obesidade; 2 - Arritmias, 3 - Doença cardíaca preexistente, irradiação mediastinal, predisposição genética; 4 - Inatividade física; 5 - Hipertensão, diabetes, dislipidemia, tabagismo; 6 - Tempo longo de terapia, dose cumulativa; 7 - Associação das classes dos quimioterápicos.

Os artigos foram identificados com a letra A, seguida do número identificador (1,2,3,4 e assim sucessivamente), de acordo com a ordem em que foram encontrados nas bases de dados.

Quadro 2 - Categorização dos artigos selecionados de acordo com os fatores de risco, tipo de câncer e classe de quimioterápicos identificados relacionados à terapia oncológica cardiotóxica.

\begin{tabular}{|c|c|c|c|c|}
\hline ARTIGOS & CATEGORIAS & FATOR DE RISCO & $\begin{array}{l}\text { TIPO DE } \\
\text { CÂNCER }\end{array}$ & $\begin{array}{c}\text { CLASSE DE } \\
\text { QUIMIOTERÁPICOS }\end{array}$ \\
\hline $\begin{array}{l}\text { A1 } \\
\text { A12 }\end{array}$ & Categoria 1 & Sobrepeso obesidade & Mama & Antraciclinas trastuzumabe \\
\hline A9 & Categoria 2 & Arritmias & Mama & $\begin{array}{l}\text { Antraciclinas } \\
\text { Trastuzumabe }\end{array}$ \\
\hline $\begin{array}{l}\text { A3 } \\
\text { A4 } \\
\text { A10 } \\
\text { A20 } \\
\text { A23 }\end{array}$ & Categoria 3 & $\begin{array}{l}\text { Doença cardíaca } \\
\text { preexistente } \\
\text { Irradiação mediastinal } \\
\text { Predisposição genética }\end{array}$ & $\begin{array}{l}\text { Mama } \\
\text { Linfoma de } \\
\text { Hodgkin e } \\
\text { linfoma não- } \\
\text { Hodgkin }\end{array}$ & Antraciclinas \\
\hline $\begin{array}{c}\text { A5 } \\
\text { A13 }\end{array}$ & Categoria 4 & Inatividade física & Mama & Antraciclinas \\
\hline $\begin{array}{c}\text { A2 } \\
\text { A7 } \\
\text { A8 } \\
\text { A11 } \\
\text { A14 } \\
\text { A17 } \\
\text { A18 }\end{array}$ & Categoria 5 & $\begin{array}{l}\text { Hipertensão, diabetes, } \\
\text { dislipidemia, tabagismo }\end{array}$ & Mama & $\begin{array}{c}\text { Trastuzumabe } \\
\text { Antraciclinas } \\
\text { Lapatinibe }\end{array}$ \\
\hline $\begin{array}{l}\text { A } 6 \\
\text { A15 } \\
\text { A19 } \\
\text { A } 21 \\
\text { A22 }\end{array}$ & Categoria6 & $\begin{array}{c}\text { Tempo longo de terapia } \\
\text { Dose cumulativa }\end{array}$ & Mama & $\begin{array}{c}\text { Trastuzumabe } \\
\text { Antracilina }\end{array}$ \\
\hline A16 & Categoria 7 & $\begin{array}{c}\text { Associação das classes dos } \\
\text { quimioterápicos }\end{array}$ & Mama & Trastuzumabe Antraciclina \\
\hline
\end{tabular}

Fonte: Dados da pesquisa. 
O Quadro 2 apresenta os principais fatores de risco descritos nos estudos, bem como a associação com os alguns tipos de câncer, sendo o de mama o mais prevalente no contexto da busca. Outro fator a ser considerado diz respeito à classe de quimioterápicos com risco para a cardiotoxicidade, sendo eles trastuzumabe, antraciclina e lapatinibe.

\section{Discussão}

\section{Categoria 1-Sobrepeso e obesidade (A1, A12)}

Os artigos pertencentes a esta categoria são referentes ao fator de risco sobrepeso e obesidade. O estudo A1, observacional do tipo coorte, utilizou dados prospectivos. Teve como cenário 26 centros de oncologia e acompanhou929 pacientes com diagnóstico de câncer de mama tratados com antraciclinas e trastuzumabe. O objetivo do estudo foi analisar a associação do índice de massa corporal com a cardiotoxicidade. Demonstrou-se que a obesidade pode estar fortemente associada à cardiotoxicidade. No estudo, compararam pacientes com peso normal e indivíduoscom sobrepeso e obesos,sendo a cardiotoxicidade mais evidenciada em indivíduos com sobrepeso e obesos (G. Kaboréet al., 2019).

O estudo A12, de caráter prospectivo e randomizado,sugere que o trastuzumabe pode melhorar os resultados de pacientes diagnosticados com câncer de mama, no entanto enfatiza que o medicamento tem forte associação com toxicidade cardíaca. Além disso, o estudo aponta o risco significativo do sobrepeso e a obesidade para o desenvolvimento da toxicidade cardíaca, principalmente em esquemas conjunto de trastuzumabe e antraciclina (Goriet al., 2018).

\section{Categoria 2 -Arritmias (A9)}

O estudo A9, uma revisão sistemática com meta-análise, utilizou a pesquisa bibliográfica para investigar a incidência da fibrilação atrial (FA) em pacientes com câncer de mamaque receberam como forma de tratamento o trastuzumabe. Nos seus resultados,observou-se uma discreta incidência de FA, e a progressão do câncer e da terapia antineoplásica pode aumentar o risco de desenvolvimento dessa arritmia (Yuan et al., 2018).

Além disso, a classe das antraciclinas são os quimioterápicos mais usados na terapia contra o câncer, sendo claramente a classe mais relacionada à toxicidade cardíaca, inclusive no risco de arritmias (Yaylali et al., 2016).Produz disfunção no sistema de condução, interrompendo uma via molecular, causando uma arritmia específica, ou provocando danos ao tecido do miocárdio, como fibrose, isquemia, apoptose, reação imunológica, inflamação, distúrbio de eletrólitos, metabólicos e endócrinos (Sánchez \&Milián, 2020).

\section{Categoria 3 - Doença cardíaca preexistente, irradiação mediastinal e predisposição genética (A3, A4, A10, A20, A23)}

O estudo A3, um ensaio clínico randomizado,ressaltou o impacto do treinamento físico na cardiotoxicidade em mulheres com câncer de mama em uso de antraciclina. Além disso, mencionou a dose cumulativa, doenças preexistentes e irradiação mediastinal como um importante fator de risco (Antunes et al., 2019).

Em relação ao artigo A4, estudo de revisão sistemática com metanáliseem um total de 186 pacientes com câncer de mama, linfoma de Hodgkine linfoma não-Hodgkin tratados com antraciclinas teve por objetivo avaliar se o estado basal de tiol/dissulfeto pode predizer a ocorrência de toxicidade cardíaca induzida por antraciclina. Em seus achados, os autores identificaram que pacientes que possuem um nível de dissulfeto mais alto ou nível de tiol de linha de base mais baixo pode indicar que está mais propício a alterações estruturais e celulares cardíacas (Bergamini et al., 2019).

Já o A10, estudo de coorte prospectivo realizado em um centro de pacientes com câncer, analisou mulheres em uso de trastuzumabe. O objetivo foi questionar o uso do trastuzumabe em pacientes com função cardíaca basal reduzida. Em seu 
resultado, constatou-se que a fração de ejeção não é reduzida quando se utiliza o trastuzumabe como opção de tratamento, entretanto o desenvolvimento da insuficiência cardíaca sintomática é mais frequente (Nowsheenet al., 2018).

$\mathrm{O}$ artigo A20 menciona paciente em estágio inicial de câncer de mama em tratamento com antraciclina e taxano com ou sem trastuzumabe. O ensaio clínico randomizado descrevequemulheres sobreviventesao câncer de mama e que não possuem história cardíaca prévia quando o trastuzumabe é incorporado na terapia adjuvante com antraciclina não têm repercussão cardíaca em longo prazo (Ganz et al., 2010).

Em um relato de caso (A23) de uma paciente com diagnóstico de câncer de mama queapresentou sinais clínicos de insuficiência cardíaca grave, sugeriu-se uma associaçãoempacientes em uso de antraciclinas com mutações no gene LMNA parapredisposição genética à cardiomiopatia induzida (Kuruc et al., 2019).

De acordo com a revista da Sociedade Portuguesa de Cardiologia, pacientes com a história de doença cardíaca preexistente apresentam uma maior incidência de surgimento de efeitos cardiotóxicos, comprovando esses achados (Adão et al., 2013).

Segundo Bittar e Fonseca (2017), a radiação mediastinal se configura como uma das principais preocupações referentes à toxicidade durante o tratamento. $\mathrm{O}$ autor enfatiza também que a doença cardíaca induzida pela radioterapia pode comprometer qualquer estrutura cardíaca, como pericárdio, miocárdio, valvas, sistema de condução e coronárias. Além disso, a própria radiação intensificou as propriedades cardiotóxicasde alguns quimioterápicos, por exemplo, os pertencentes à classe das antraciclinas.

A própria predisposição genética influencia como fator de risco para cardiotoxicidade. Cruz et al. (2016) mencionam os fatores de risco da cardiotoxicidade induzida por antraciclina ligados à predisposição genética, sexo feminino e pacientes de raça negra.

\section{Categoria 4 - Inatividade física (A5, A 13)}

Um estudo do tipo ensaio clínico não randomizado que investigou a terapêutica do exercício físico em mulheres com câncer de mama em uso de antraciclinae que teve por objetivo avaliar a relação da quimioterapia com a aptidão cardiopulmonar demonstrou em seus resultados que pacientes em uso de quimioterápico obtiveram prejuízo progressivo da função cardíaca, associando a quimioterapia à diminuição da aptidão cardiopulmonar durante o exercício. Além disso, enfatizou a inatividade física como um preditor de risco da cardiotoxicidade (STEPHEN J et al., 2019). Corroborando, Dessalviet al. (2018) mencionam o exercício físico como um fator protetor direcionado aos fatores de risco cardiovascular.

Sugere-se que a atividade física como treinamento aeróbico e de força muscular está relacionada à diminuição de efeitos negativos dos quimioterápicos, principalmente referentes ao sistema cardiovascular (Corrêa et al., 2019), sendo um importante cardioprotetor e adjunto de estratégias para a segurança cardíaca nos pacientes oncológicos em tratamento.

\section{Categoria 5 - Hipertensão, diabetes, dislipidemia, tabagismo (A2, A7, A8, A11, A14, A17, A18)}

Uma revisão sistemática A2 publicada em setembro de 2019 relacionou a toxicidade induzida por quimioterapia à base de antraciclina. Neste estudo, os autores identificaram os fatores risco de cardiotoxicidade, dentre eles risco de desenvolvimento de insuficiência cardíaca, caracterizado pela redução da FE, pressão arterial basal moderadamente elevada e tempo de contração isovolumétrico aumentado (Lin \& Lengacher, 2019).

O estudo A7, do tipo observacional, prospectivo, multicêntrico e longitudinal ,realizado com 41 mulheres com câncer de mama tratadas com trastuzumabe, elencou em seus resultadosos fatores de risco das participantes do estudo. A hipertensão eo tabagismoforamos fatores mais presentes, o diabetes e a dislipidemia assumiram a terceira e quarta opções, respectivamente, em último a doença coronariana (Gong et al., 2018). 
Revisão sistemática (A8), com o objetivo de revisar o risco de toxicidades cardiovasculares, sugeriu um significativo risco de toxicidade cardíaca em pacientes com câncer tratados com inibidores da tirosina quinase do receptor do fator de crescimento endotelial vascular (Li e Gu, 2018).

O estudo A11, de revisão, registrou a relevância da avaliação pré-quimioterápica focada nos fatores de risco cardiovascular, sobretudo a avaliação da medição da pressão arterial (Sharalaya \& Collier, 2018).

A revisão (A14) teve como objetivo resumir o efeito cardiotóxico induzido por Everolimus. Identificou-se que o único efeito adverso relacionado a essa terapia é indireto. Entretanto, estão diretamente associados a fatores de risco cardiovasculares como hiperglicemia, hiperlipidemia e hipertensão (Karvelas et al., 2018).

Já o estudo A17, randomizado e multicêntrico, avaliou lapatinibe e trastuzumabe em pacientes com câncer de mama, tendo como objetivo a avaliação dos níveis de biomarcadores cardíacos TnT e NTproBNP após semanas de quimioterapia.Um dos seus resultados demonstrou que pacientes com histórico de hipertensão apresentavam mais alterações de biomarcadores cardíacos comparados àqueles indivíduos com níveis pressóricos normais.Além disso, um paciente diabético igualmente apresentou alterações de biomarcadores (Ponde et al., 2017).

O artigo A18, um estudo piloto realizado entre o ano de 2013 e 2017, em três centros nos EUA,avaliou a segurança cardíaca do trastuzumabe em 30 pacientes com disfunção cardíaca. Em seus resultados, levantaram-se os fatores associados à cardiotoxicidade, como sobrepeso $(32,3 \%)$ /obeso $(51,6)$, hipertensão $(4,9)$, diabetes $(16,1)$ e dislipidemia $(38,7)$ (Lynce et al., 2019).

Condições como hipertensão, fatores de risco cardiovasculares preexistentes e dislipidemia têm forte ligação no impacto do risco da cardiotoxicidade relacionado ao uso da quimioterapia, em especial quimioterápicos da classe das antraciclinas (Reinbolt et al., 2015). Gavilaet al. (2017) ressaltam, além da hipertensão, o tabagismo, idade, diabetes e sedentarismo como fatores vulneráveis à toxicidade cardíaca.

\section{Categoria 6 - Tempo longo de terapia e Dose cumulativa (A6, A15, A19, A21, A22)}

O estudo (A6), revisão sistemática, objetivou identificar estudos que comparavam a duração do tratamento com trastuzumabe em um ano e em um menor período em pacientes com diagnóstico de câncer de mama. $\mathrm{O}$ estudo identificou que pacientes com câncer de alto risco que fazem uso da terapia durante um ano possuem melhor desfecho no tratamento, no entanto os eventos cardíacos foram significativamente menores em pacientes que fizeram a terapia quimioterápica em um período mais curto que um ano (Inno et al., 2018).

Já no artigo A15, ensaio controlado, duplo cego, com 69 mulheres tratadas com terapia adjuvante do câncer de mama, testou-se a hipótese de que o tratamento adjuvante para o câncer de mama precoce com a antraciclina e epirrubicina está associado à dose dependente do aumento da fração de extração do volume extravascular. A cardiotoxicidade provocada por antraciclinas depende da dose e aumentou a fração de extração de volume extravascular. Os pacientes que receberam candesartan apresentaram menos declínio na função sistólica do que os pacientes que não receberam candesartan (Hecket al., 2018).

Outro artigo encontrado (A19), estudo randomizado e multicêntrico, comparou o efeito do tratamento com trastuzumabe, utilizado com duração de um e dois anos de diferença. Em seus resultados, não houve evidências de um benefício adicional de um segundo ano de trastuzumabe, mas existem algumas evidências de toxicidade cardíaca adicional com maior duração do tratamento (Cameron et al., 2017).

Um estudo randomizadoe multicêntrico (A21) procurou comparar a efetividade do trastuzumabe utilizado durante nove semanas versus a utilização durante um ano. Os resultados mostraram a melhor efetividade do quimioterápico utilizado 
por um ano. Contudo, a administração do trastuzumabe por nove semanas diminuiu o risco de toxicidade cardíaca e pode ser uma opção para pacientes com risco cardíaco e baixo risco de recidiva (Conte et al., 2018).

Já o artigo A22, um estudo multicêntrico Delphi, envolvendo 100 oncologistas, destacou que a dose cumulativa de antraciclinas em combinação com outras drogas é um fator de risco e importante preditor para cardiotoxicidade, podendo aumentar a vulnerabilidade cardiovascular e relação direta com a insuficiência cardíaca (Gavilaet al., 2016).

As doses cumulativas de antraciclinas têm relação direta com a presença de insuficiência cardíaca.Ela recomenda que o uso de antraciclina deva ser reduzido, mantendo uma dose cumulativa menor que o limite recomendado ao longo da vida do paciente em tratamento oncológico (Gavilaet al., 2016).

\section{Categoria7- Associação das classes dos quimioterápicos}

O estudo não randomizado (A16), de fase II, aberto, multicêntrico e multinacional em pacientes com função cardíaca normal, com o objetivo de avaliar a segurança cardíaca do trastuzumabe, pertuzumabe em oito ciclos de quimioterapia neoadjuvante,mostrou que tratamento com pertuzumabe, trastuzumabe e regimes de quimioterapia contendo antraciclina, comum para o tratamento neoadjuvante, resultou em perfis cardíacos e de segurança geral. Todavia, o trastuzumabe está associado a um risco de toxicidade cardíaca, principalmente quando administrado com antraciclinas (Swainet al., 2018).

Os agentes antineoplásicos cardiotóxicos são classificados em duas particularidades. Tipo I, fármacos pertencentes à classe das antraciclinas, dependente da dose utilizada, são responsáveis por gerar lesões irreversíveis. E do tipo II, pertencentes à classe dos trastuzumabe, que não dependem da dose utilizada e geralmente seus efeitos são reversíveis após o final ou interrupção do tratamento (Cruz et al., 2016).

As modalidades terapêuticas, como a classe das antraciclinas,e os anticorpos monoclonais, como o trastuzumabe, são os antineoplásicos mais utilizados como forma de tratamento do câncer. Representa uma das classes mais conhecidas por induzir a cardiotoxicidade (Cruz et al., 2016).

Ratificando, para Reinboltet al.(2015), os efeitos prejudiciais advindos dos quimioterápicos pertencentes às antraciclinas podem ser potencializados por outros medicamentos, particularmente o anticorpo monoclonal trastuzumabe (Reinbolt et al., 2015).

Sendo assim, a cardio-oncologia revela-se com uma nova subespecialidade, emergiu da intercessão da cardiologia e a oncologia. Nos últimos anos, houve uma mudança epidemiológica, na medida em que a doença oncológica vem assumindo destaque e liderança, sendo a principal causa de morte da população, ultrapassando as doenças cardiovasculares, de acordo com dados epidemiológicos dos Estados Unidos (Heron \& Anderson, 2016).

Nos últimos anos vem sendo um assunto de interesse para muitos profissionais da saúde, dada as complicações cardiovasculares observadas em pacientes oncológicos. A cardiotoxicidade é um dos eventos adversos mais expressivos durante o tratamento oncológico, sendo responsável pelas complicações cardiovasculares no paciente com câncer e pode se apresentar de forma aguda, subaguda ou crônica. As formas agudas e subagudas usualmente aparecem no início do tratamento, até duas semanas depois de finalizada a terapêutica,sendo expressas por alterações de repolarização ventricular, variações no intervalo QT, arritmias, síndromes coronarianas agudas, infecções do pericárdio e miocárdio (Diretriz Brasileira de CardioOncologia da Sociedade Brasileira de Cardiologia, 2020 2011) (Adão et al., 2013).

A condição crônica possui dois grupos e se diferencia através do início dos sintomas clínicos. O primeiro subtipo é caracterizado por ocorrer no primeiro ano após o término do tratamento quimioterápico, e o segundo subtipo, acontece após um ano do término. A manifestação mais presente da forma crônica é a disfunção ventricular sistólica ou diastólica, podendo induzir a insuficiência cardíaca e apoptose cardiovascular (Diretriz Brasileira de Cardio-Oncologia da Sociedade Brasileira de Cardiologia, 2020). 


\section{Considerações Finais}

No presente trabalho, explanaram-se os principais fatores de riscos associados à cardiotoxicidade e as principais classes de quimioterápicos responsáveis pelo efeito cardiotóxico, sendo eles o sobrepeso e obesidade, arritmias cardíacas, doença cardíaca preexistente, irradiação mediastinal, predisposição genética, inatividade física, hipertensão, diabetes, dislipidemia, tabagismo, tempo longo de terapia, dose cumulativa e associação das classes dos quimioterápicos (antraciclinas, anticorpos monoclonais e inibidores da tirosina quinase).

Os efeitos cardiotóxicos do tratamento quimioterápico podem ser reversíveis e irreversíveis. Por esse motivo, a elaboração de estratégias para prevenção e redução desses efeitos é extremamente importante, no sentido de melhorar o prognóstico e qualidade de vida do paciente oncológico.

Entende-se que a prevenção da cardiotoxicidade deverá iniciar antes do início do tratamento quimioterápico. Os pacientes tratados com drogas cardiotóxicas devem ser acompanhados continuamente antes, durante e após o início do seu tratamento. O rastreio dos fatores de risco e o seu controle tornam-se essenciais, uma vez que existem fatores de risco modificáveis, tais como hipertensão, sobrepeso, dislipidemia, tabagismo,passíveis de monitoramento e manejo pelo enfermeiro.

No decorrer da revisão, pode-se observar a carência de pesquisas relacionadas à cardiotoxicidade no âmbito da enfermagem de origem nacional, entretanto são encontrados artigos de origem médica em revistas internacionais. Nessa perspectiva, surge a necessidade da realização de mais pesquisas na área de Enfermagem que abordem o contexto dos fatores de risco na cardiotoxicidade, visto que, é o enfermeiro quem acompanha continuamente o paciente em todo o ciclo quimioterápico. A carência de estudos abordando essa temática deixa como sugestão para realização de novas pesquisas.

O estudo pretendeu contribuir com a prática da enfermagem que atua com essa população, possibilitando a reflexão sobre os fatores de risco capazes de predizer e facilitar o desenvolvimento da cardiotoxicidade e as principais drogas que podem induzir a toxicidade. Além de enfatizar a importância da vigilância cardiovascular contínua no paciente oncológico em tratamento antineoplásico.

\section{Referências}

Adão, R., et al (2013). Cardiotoxicidadeassociadaàterapê uticaoncológica:mecanismosfisiopatológicoseestratégiasdeprevenc ?ão. Revista Portuguesa de Cardiologia, 32(5), 395-409. https://doi.org/x.doi.org/10.1016/j.repc.2012.11.002

Antunes, P., et al (2019). Impact of exercise training on cardiotoxicity and cardiac health outcomes in women with breast cancer anthracycline chemotherapy: a study protocol for a randomized controlled trial. Trials, 20(1), 1-11. https://doi.org/doi.org/10.1186/s13063-019-3499-9

Bergamini, C., et al (2019). Role of Speckle Tracking Echocardiography in the Evaluation of Breast Cancer Patients Undergoing Chemotherapy: Review and Meta-analysis of the Literature. Cardiovascular Toxicology, 19(6), 485-492. https://doi.org/10.1007/s12012-019-09523-y

Bittar, Cristina Salvadori , \& Fonseca, Silvia Moulin Ribeiro . (2017, November). RADIOTERAPIA E CARDIOTOXICIDADE. Rev Soc Cardiol Estado de São Paulo, 4(29), 274-277. https://doi.org/http://dx.doi.org/10.29381/0103-8559/20172704274-7

Cameron, D., et al. (2017). 11 years' follow-up of trastuzumab after adjuvant chemotherapy in HER2-positive early breast cancer: final analysis of the HERceptin Adjuvant (HERA) trial. The Lancet, 389(10075), 1195-1205.

Conte, P., Frassoldati, A, Bisagni, G., Brandes, A. A., Donadio, M., Garrone, Ó., Piacentini, F., Cavanna, L., Giotta, F., Aieta, M., Gebbia, V., Molino, A., musolino, U. M., Ferro, A., Maltoni, R., Danese, S., Zamagni, C., Rimanti , A., Cagossi, K., Russo, U. M., Pronzato, P., Giovanardi, F., Moretti, G., Lombardo, L., Schirone, A., Beano, A., Amaducci, L., Bajardi, E. A., Vicini , R., Balduzzi, S , D'Amico , R., \& Guarneri, V . (2018). Nine weeks versus 1 year adjuvant trastuzumab in combination with chemotherapy: final results of the phase III randomized Short-HER study. Annals Of Oncology, 29(12), 23282333. https://doi.org/10.1093/annonc/mdy414

Corrêa, M. V. dos S. , Chermont, S. L. S. M. da C., Marinho, T. A. S., \& Quintão, M. M. P. (2019). Importância da Prática de Atividade Física para Prevenção do Risco de Cardiotoxicidade: Revisão Sistemática. Revista Brasileira de Cancerologi, 65(3), 1-10. https://doi.org//doi.org/10.32635/21769745.RBC.2019v65n3.433

Cruz, M., Rodrigues, J. D., \& Campelo, M. (2016). Cardiotoxicidadenaterapêuticacomantraciclinas:estratégiasdeprevenc ?ão. Revista Portuguesa de Cardiologia, 35(6), 359-371. https://doi.org/x.doi.org/10.1016/j.repc.2015.12.004 
Departamento de Atenção Básica., Ministério da Saúde. Secretaria de Atenção à Saúde. (2013). Diretrizes para o cuidado das pessoas com doenças crônicas nas redes de atenção à saúde e nas linhas de cuidado prioritárias. Ministério da Saúde. https://bvsms.saude.gov.b r/bvs/publicacoes/diretrizes\%20_cuidado_pessoas\%20_doencas_cronicas.pdf

Dessalvi, C. C., et al (2018). Chemotherapy-induced cardiotoxicity: new insights into mechanisms, monitoring, and prevention. Journal Of Cardiovascular Medicine, 19(7), 315-323. https://doi.org/10.2459/JCM.0000000000000667

Diretriz Brasileira de Cardio- Oncologia da Sociedade Brasileira de Cardiologia. (2020). Sociedade Brasileira de Cardiologia, 1-38. http://abccardiol.org/wpcontent/uploads/articles_xml/1678-4170-abc-115-05-1006/1678-4170-abc-115-05-1006.x64000.pdf

Estimativa 2020: incidência de câncer no Brasil / Instituto Nacional de Câncer. (2019). INCA. https://www.inca.gov.br/sites/ufu.sti.inca.local/files/media/document/estimativa-2020-incidencia-de-cancer-no-brasil.pdf

European Heart Journal. (2016). ESC Position Paper on cancer treatments and cardiovascular toxicity developed under the auspices of the ESC Committee for Practice Guidelines: ?The Task Force for cancer treatments and cardiovascular toxicity of the European Society of Cardiology (ESC), 2768-2801. https://academic.oup.com/eurheartj/article/37/36/2768/2197413

Lynce, F., Barac, A., Geng, X., Dang, C., Yu, A., Smith, K., G allagher, C., Pohlmann, P., Nunes, R., \& Herbolsheimer, P.Prospective evaluation of the cardiac safety of HER2-targeted therapies in patients with HER2-positive breast cancer and compromised heart function: the SAFE-HEaRt study. Breast Cancer Research And Treatment, 175(3), . 595-603. https://doi.org/doi: 10.1007 / s10549-019-05191-2.

Folha informativa ? Câncer. (2018). OPAS BRASIL. https://www.paho.org/bra/index.php?option=com_content\&view=article\&id=5588:folha-informativacancer\&Itemid=1094

G. Kaboré , E., et al. (2019). Association of body mass index and cardiotoxicity related to anthracyclines and trastuzumab in early breast cancer: French CANTO cohort study. Plos Medicine, 16(12), 1-12. https://doi.org/doi.org/10.1371/journal.pmed.1002989 D

Ganz, P. A., et al (2010). Long-Term Follow-Up of Cardiac Function and Quality of Life for Patients in NSABP Protocol B-31/NRG Oncology: A Randomized Trial Comparing the Safety and Efficacy of Doxorubicin and Cyclophosphamide (AC) Followed by Paclitaxel With AC Followed by Paclitaxel and Trastuzumab in Patients With Node-Positive Breast Cancer With Tumors Overexpressing Human Epidermal Growth Factor Receptor 2. Journal Of Clinical Oncology, 35(35), 3942-3948. https://doi.org/DOI: https://doi.org/10.1200/JCO.2017. 74.1165

Gavila, J., Segui, M. A., Calvo, L., López, T., Alonso, J. J., Farto, M., \& Rosa, R., Sánchez-de la . (2016). Evaluation and management of chemotherapyinduced cardiotoxicity in breast cancer: a Delphi study. Clinical And Translational Oncology, 19(1), 91?104. https://doi.org/DOI 10.1007/s12094-016-1508-y

Gong, I. Y., et al. Early diastolic strain rate measurements by cardiac MRI in breast cancer patients treated with trastuzumab: a longitudinal study. The International Journal Of Cardiovascular Imaging, 35(4), 653-662. https://doi.org/DOI: 10.1007/s10554-018-1482-2

Gori, S., et al. When and how to treat women with HER2-positive, small (pT1a-b), nodenegative breast cancer? Critical Reviews In Oncology/hematology, 128, 130-138. https://doi.org/doi.org/10.1016/j.critrevonc.2018.03.010

Heck, S. L., et al (2018). Effect of candesartan and metoprolol on myocardial tissue composition during anthracycline treatment: the PRADA trial. European Heart Journal - Cardiovascular Imaging, 19(5), 544-552. https://doi.org/10.1093/ehjci/jex159

Heron, M., \& Anderson, R. N. (2016, August). Mudanças na causa principal de morte: padrões recentes em Doença cardíaca e mortalidade por câncer. de Controle e Prevenção de Doenças, 254, 1-8. file:///D:/Meus\%20Documentos/Downloads/dados\%20epidemiol\%C3\%B3gicos.en.pt.pdf

Inno, A., et al.(October). One year versus a shorter duration of adjuvant trastuzumab for HER2-positive early breast cancer: a systematic review and metaanalysis. Breast Cancer Research And Treatment, 173(2), 247-254. https://doi.org/10.1007/s10549-018-5001-x

Karvelas, G., et al. (July). Everolimus as cancer therapy: Cardiotoxic or an unexpected antiatherogenic agent? A narrative review. Hellenic Journal Of Cardiology, 59(4), 196-200.

Köche, J. K. (2011). Fundamentos de metodologia científica: teoria da ciência e iniciação à pesquisa (ed. Original). Vozes. http://www.brunovivas.com/wpcontent/uploads/sites/10/2018/07/K\%C3\%B6che-Jos\%C3\%A9-Carlos0D0AFundamentos-de-metodologia-cient\%C3\%ADfica-_- teoria-da0D0Aci\% C3\% AAncia-e-inicia\% C3\% A7\% C3\% A3o-\% C3\% A0-pesquisa.pdf

Kuruc, J. C., Archibold, A. A. D., Motta, J., Rao, K. S., Trachtenberg, B., Ramos, C., Wang, H., Gorenstein, D., Vannberg, F., \& Jordan, K. (2019). Desenvolvimento de cardiomiopatia dilatada induzida por antraciclina por mutação no gene LMNA em paciente com câncer de mama: relato de caso. BMC Cardiovascular Disorders , 19 (1), 1-5. https://doi.org/10.1186/s12872-019-1155-7

Li, J., \& Gu, J. (2018). Cardiovascular Toxicities with Vascular Endothelial Growth Factor Receptor Tyrosine Kinase Inhibitors in Cancer Patients: A MetaAnalysis of 77 Randomized Controlled Trials. Clinical Drug Investigation, 38(12), 1109-1123. https://doi.org/10.1007/s40261-018-0709-2

Lin, K., \& Lengacher, C. A . (2019). Anthracycline Chemotherapy-Induced Cardiotoxicity in Breast Cancer Survivors: A Systematic Review. Oncol Nurs Forum, 46(5), 145-158. https://doi.org/10.1188/19.ONF.E145-E158

Lins de Barros, M. V., et al (2018). Left Ventricular Regional Wall Motion Abnormality is a Strong Predictor of Cardiotoxicity in Breast Cancer Patients Undergoing Chemotherapy. Arquivos Brasileiros de Cardiologia, 50-56. https://doi.org/10.5935/abc.20180220

Nowsheen, S., et al (2018). Trastuzumab in Female Breast Cancer Patients With Reduced Left Ventricular Ejection Fraction. Journal Of The American Heart Association, 7(15), 1-12. https://doi.org/doi: 10.1161/JAHA.118.008637

Pereira, A. S., Shitsuka, D. M., Parreira, F. J, \& Shitsuka, R. (2018). Metodologia da Pesquisa Científica. UAB / NTE / UFSM. https://repositorio.ufsm.br/bitstream/handle/1/15824/Lic_Computacao_Metodologia-Pesquisa-Cientifica.pdf?sequence=1 
Research, Society and Development, v. 10, n. 2, e9110212299, 2021 (CC BY 4.0) | ISSN 2525-3409 | DOI: http://dx.doi.org/10.33448/rsd-v10i2.12299

Pina, L. C. de O., et al. Cardiotoxicidade nas Terapias Neoadjuvante e Adjuvante do Câncer de Mama. Revista Brasileira de Cancerologia, 65(3), 1-8. https://doi.org/doi: https://doi.org/10.32635/2176-9745.RBC.2019v65n3.404

Ponde, N., et al. Cardiac biomarkers for early detection and prediction of trastuzumab and/or lapatinib-induced cardiotoxicity in patients with HER2-positive early-stage breast cancer: a NeoALTTO sub-study (BIG 1-06)., 168(3), 631-638.

Reinbolt , R. E., et al. (2015). Risk factors for anthracycline-associated cardiotoxicity. Supportive Care In Cancer, 24(5), 2173-2180. https://doi.org/doi: $10.1007 / \mathrm{s} 00520-015-3008-y$

Sánchez, M. D., \& Milián, M. B. (2020). Sinais elétricos de arritmias ventriculares malignas e morte súbita .: importância em pacientes recebendo terapia anticâncer. Revista Cubana do Cardiologia y Cirurgia Cardiovascular, 23(15), 1-5. file:///D:/Meus\%20Documentos/Downlo ads/arrtimia\%20fator\%20de\%20risco.es.pt.pdf

Sharalaya, A., \& Collier, P. (2018). Prevention of Cardiotoxicities With Traditional and Novel Chemotherapeutic Agents. Current Heart Failure Reports, 5(4), 260-269. https://doi.org/DOI: 10.1007/s11897-018-0400-1

Siewert, J. S., Rodrigues, D. B., de Malfussi, L. B. H., de Andrade, S. R., \& Erdmann, A. L. (2017). Gestão do Cuidado Integral em Enfermagem: Reflexões sob a Perspectiva do Pensamento Complexo. Revista Mineira de Enfermagem , 21 , 1-5.

Stephen J., ey al (2019). Persistent Impairment in Cardiopulmonary Fitness after Breast Cancer Chemotherapy. Medicine \& Science In Sports \& Exercise, 51(8), 1573-1581. https://doi.org/doi: 10.1249/MSS.0000000000001970

Swain, S. M., et al (2018). Pertuzumab, trastuzumab, and standard anthracycline- and taxane-based chemotherapy for the neoadjuvant treatment of patients with HER2-positive localized breast cancer (BERENICE): a phase II, open-label, multicenter, multinational cardiac safety study. Annals Of Oncology, 29(3), 646-653. https://doi.org/10.1093/annonc/mdx773

Turato, Egberto Ribeiro. (2019). Tratado da pesquisa clínico- qualitativa: contrução teórico-epistemológica, discussão comparada e aplicação nas áreas de saúde e humana. ( $3^{\mathrm{a}}$ reimpressão). Vozes.

Yaylali , Y. T., et al (2016). Atrial Function in Patients with Breast Cancer After Treatment with Anthracyclines. Arquivos Brasileiros de Cardiologia, 411419. https://doi.org/10.5935/abc.20160146

Yuan, M., et al (2018). The incidence of atrial fibrillation with trastuzumab treatment: A systematic review and meta-analysis. Cardiovascular Therapeutics, 36(6), 12475-1280. https://doi.org/10.1111/1755-5922.12475 